Brief paper

\title{
Synthesis of Razumikhin and Lyapunov-Krasovskii approaches to stability analysis of time-delay systems ${ }^{\text {स }}$
}

\author{
Irina V. Medvedeva ${ }^{1}$, Alexey P. Zhabko \\ Applied Mathematics and Control Processes Department, St.-Petersburg State University, St.-Petersburg, Russia
}

\section{A R T I C L E I N F O}

\section{Article history:}

Received 26 June 2013

Received in revised form

6 August 2014

Accepted 2 September 2014

Available online 6 November 2014

\begin{abstract}
A B S T R A C T
In this paper, a necessary and sufficient condition for the exponential stability of linear systems with several time-delays is presented. Such a condition is based on the construction of quadratic lower bounds for the Lyapunov-Krasovskii functionals on the special Razumikhin-type set of functions. The result reveals a constructive procedure for the stability analysis whose application is illustrated with examples.
\end{abstract} (C) 2014 Elsevier Ltd. All rights reserved.

Keywords:

Time-delay system

Stability

Stability domains

Lyapunov-Krasovskii functionals

\section{Introduction}

In this paper, we analyze the stability of linear time-invariant differential systems with several delays within the framework of the Lyapunov-Krasovskii functionals. The basic concepts of the Lyapunov-Krasovskii approach and, in particular, the structure of the functionals used, were first established in Repin (1965), Krasovskii (1956) and further developed in Huang (1989), Infante and Castelan (1978) and Kharitonov and Zhabko (2003). More precisely, in Kharitonov and Zhabko (2003) the so-called complete-type functionals were introduced. These functionals admit quadratic lower and upper bounds, and for this reason, at the present time, they are effectively used for construction of exponential estimates of solutions (Kharitonov, 2013), for the stability (Egorov \& Mondie, 2013) and the robust stability analysis with respect to uncertainties in coefficients (Kharitonov, 2013) or in delay (Kharitonov \& Niculescu, 2003), for computation of critical delays (Ochoa, Mondie, \& Kharitonov, 2009). The book of Kharitonov (2013) gives a detailed survey on the present state of the art in the area.

\footnotetext{
The work was supported by Saint-Petersburg State University, the research grant 9.37.157.2014. The material in this paper was partially presented at the 11th IFAC Workshop on Time-Delay Systems (TDS), February 4-6, 2013, Grenoble, France. This paper was recommended for publication in revised form by Associate Editor Maria Elena Valcher under the direction of Editor Roberto Tempo.

E-mail addresses: medv.irina@gmail.com (I.V. Medvedeva), zhabko@apmath.spbu.ru (A.P. Zhabko).

1 Tel.: +7 9117690087 .
}

A different modification of the approach is considered in this paper. Following the work of Huang (1989), we use a functional with time derivative given as a negative definite quadratic form of the present state of the system. To apply this functional to stability analysis, one needs to obtain a quadratic lower bound on it. However, in Huang (1989) this functional is shown to admit only a local cubic lower bound on the set of solutions of the system. As a result, it is considered not to be effective in the stability analysis and applications.

On the contrary, in this contribution we propose a constructive method for stability analysis using the functional studied in Huang (1989). The idea of the method is to use a special Razumikhintype condition (see Razumikhin, 1956) for the estimation of the functional. It turns out that for the stability analysis it is sufficient to construct a lower bound for the functional on the set of functions satisfying this condition instead of the set of solutions. This enables us to establish a necessary and sufficient condition for the exponential stability, and opens a constructive way for analysis. The proposed method provides possibility to compute the critical delays (see Medvedeva \& Zhabko, 2013) and to perform the robustness analysis, as well.

The paper is organized as follows. After some preliminary definitions in Section 2, we present a constructive necessary and sufficient condition for the exponential stability (Theorem 1) in Section 3. Then, Section 4 provides a detailed exposition of the method for stability analysis which is based on the theoretical results of Section 3. In Section 5, we discuss the convergence issue (see Theorem 10) which plays a key role in the application of the method. In Section 6, we give the illustrative examples. Some concluding remarks end the paper. 


\section{Preliminaries}

We consider a time-delay system of the form

$\dot{x}(t)=\sum_{j=0}^{m} A_{j} x\left(t-h_{j}\right)$,

where $A_{j} \in \mathbb{R}^{n \times n}, j=0,1, \ldots, m$, are given constant matrices, and $0=h_{0}<h_{1}<\cdots<h_{m}=h$ are the delays. Let $t_{0}=0$ be an initial time instant, and $\varphi(\theta)$ be an initial function which is supposed to be piecewise continuous on the segment $[-h, 0]$. We denote the solution of system (1) with the initial function $\varphi$ by $x(t, \varphi)$, and the segment of the trajectory $\{x(t+\theta, \varphi) \mid \theta \in[-h, 0]\}$ by $x_{t}(\varphi)$. We omit the symbol $\varphi$ when no confusion can arise. Throughout the paper, we use the Euclidean norm for vectors, and the analogue of the uniform norm for vector functions $\|\varphi\|_{h}=\sup _{\theta \in[-h, 0]}\|\varphi(\theta)\|$.

It is well-known (see Bellman \& Cooke, 1963) that the characteristic equation of system (1) is of the form $\operatorname{det}(\lambda I-$ $\left.\sum_{j=0}^{m} A_{j} e^{-\lambda h_{j}}\right)=0$, and its roots are called eigenvalues. System (1) is said to satisfy the Lyapunov condition if it does not have the eigenvalues whose sum is equal to zero.

Given a symmetric matrix $W$, we say that $U(\tau)$ is the Lyapunov matrix associated with $W$ if it satisfies the following set of equations (see Kharitonov, 2013)

$U^{\prime}(\tau)=\sum_{j=0}^{m} U\left(\tau-h_{j}\right) A_{j}, \quad \tau \geqslant 0 ;$

$U(-\tau)=U^{T}(\tau), \quad \tau \geqslant 0 ;$

$\sum_{j=0}^{m}\left[U\left(-h_{j}\right) A_{j}+A_{j}^{T} U^{T}\left(-h_{j}\right)\right]=-W$.

The Lyapunov matrix exists and is unique for any symmetric matrix $W$, if and only if system (1) satisfies the Lyapunov condition, see Kharitonov (2013). According to Kharitonov and Zhabko (2003), the matrix $U(\tau)$ associated with $W$ determines the quadratic functional

$$
\begin{aligned}
v\left(x_{t}\right)= & x^{T}(t) U(0) x(t) \\
& +2 x^{T}(t) \sum_{j=1}^{m} \int_{-h_{j}}^{0} U\left(-\theta-h_{j}\right) A_{j} x(t+\theta) d \theta \\
& +\sum_{k=1}^{m} \sum_{j=1}^{m} \int_{-h_{k}}^{0} x^{T}\left(t+\theta_{1}\right) A_{k}^{T} \\
& \times\left(\int_{-h_{j}}^{0} U\left(\theta_{1}+h_{k}-\theta_{2}-h_{j}\right) A_{j} x\left(t+\theta_{2}\right) d \theta_{2}\right) d \theta_{1},
\end{aligned}
$$

such that its time derivative along the solutions of system (1) is equal to $-x^{T}(t) W x(t)$. One can check the last fact directly, using properties (2) of the Lyapunov matrix.

To define a critical delay, we first suppose that $h_{j}=\alpha_{j} \mathfrak{h}, j=$ $1,2, \ldots, m$, where $\alpha_{j}>0$ are fixed, and $\mathfrak{h} \geqslant 0$ is a basic delay. The values of basic delay for which system (1) changes the property of exponential stability to the instability, or vice versa, are said to be the critical delays.

Finally, let us introduce the set

$S=\{\varphi:\|\varphi(\theta)\| \leqslant\|\varphi(0)\|, \theta \in[-h, 0]\}$.

The set $S$ is the very set whereon we will further check the positive definiteness of the functional $v$.

\section{Stability theorem}

Here we present our main stability result.

Theorem 1. Given a positive definite matrix $W$, system (1) is exponentially stable, if and only if there exists a functional $v(\varphi)$ such that the following conditions hold:

1. $\frac{d v\left(x_{t}\right)}{d t}=-x^{T}(t) W x(t)$ along the solutions of system (1);

2. On the set $S$ the functional admits a lower bound of the form

$$
v(\varphi) \geqslant \mu\|\varphi(0)\|^{2}, \quad \mu>0 .
$$

Proof. Necessity. The idea of the proof of the necessity part is borrowed from Huang (1989). Let $v$ be a functional of the form (3), then the first condition of the theorem holds, as was mentioned in the previous section. To prove the second one, take an arbitrary function $\varphi \in S$, and set $\alpha=\|\varphi\|_{h}$. Since $\varphi \in S$, we have $\|\varphi(0)\|=\alpha$. Integrating system (1) and using Gronwall's lemma (see Bellman \& Cooke, 1963, p. 31), we obtain that

$\|x(t, \varphi)\| \leqslant N(t), \quad$ where $N(t)=\alpha K_{1} e^{K t}$,

$K=\sum_{j=0}^{m}\left\|A_{j}\right\|, \quad K_{1}=1+\sum_{j=1}^{m}\left\|A_{j}\right\| h_{j}$.

Hence, $\|\dot{x}(t, \varphi)\| \leqslant K N(t) \leqslant K N(\delta) \forall t \leqslant \delta, \forall \delta>0$, and $\| x(t, \varphi)-$ $\varphi(0) \| \leqslant K N(\delta) \delta, t \leqslant \delta$. Let us choose $\delta$ so that $K N(\delta)=\alpha /(2 \delta)$, note that $\delta$ does not depend on $\alpha$. Then,

$\|x(t, \varphi)\| \geqslant\|\varphi(0)\|-K N(\delta) \delta=\|\varphi(0)\| / 2, \quad t \leqslant \delta$.

Since system (1) is exponentially stable, we have

$$
\begin{aligned}
v(\varphi) & =\int_{0}^{\infty} x^{T}(t, \varphi) W x(t, \varphi) d t \\
& \geqslant \lambda_{\min }(W) \int_{0}^{\delta}\|x(t, \varphi)\|^{2} d t \geqslant \lambda_{\min }(W) \delta \frac{\|\varphi(0)\|^{2}}{4},
\end{aligned}
$$

where $\lambda_{\min }(W)$ is the smallest eigenvalue of $W$. Thus, $\mu=$ $\lambda_{\min }(W) \delta / 4>0$, and the proof of necessity is complete. Note that $\mu$ is obtained constructively.

Sufficiency. Suppose that there exists a functional of the form (3) satisfying the second condition of the theorem but system (1) is not exponentially stable. Then there exists a sequence $\left\{t_{k}\right\}_{k=1}^{\infty}$, such that $t_{k}-t_{k-1} \geqslant h, t_{k} \underset{k \rightarrow+\infty}{\longrightarrow}+\infty$, and $\left\|x\left(t_{k}\right)\right\| \geqslant \beta>0$. Consider two cases.

1. Let the solution $x(t)$ be uniformly bounded, i.e. there exists $G>0$ such that $\|x(t)\| \leqslant G \forall t \geqslant 0$. Then, $\|\dot{x}(t)\| \leqslant K G \forall t \geqslant 0$, where $K=\sum_{j=0}^{m}\left\|A_{j}\right\|$.

Take $t \in\left[t_{k}, t_{k}+\tau\right], \tau>0$. Then, $\left\|x(t)-x\left(t_{k}\right)\right\| \leqslant K G\left(t-t_{k}\right) \leqslant$ $K G \tau$, and, choosing $\tau=\min \left\{\frac{\beta}{2 K G} ; h\right\}$, we obtain

$\|x(t)\| \geqslant\left\|x\left(t_{k}\right)\right\|-K G \tau \geqslant \frac{\beta}{2}, \quad t \in\left[t_{k}, t_{k}+\tau\right]$,

for every $k$. Further, let $N(t)$ be the number of intervals $\left[t_{k}, t_{k}+\tau\right]$, contained in $[0, t]$. These intervals do not intersect with each other due to the choice of $\tau$, and $N(t) \underset{t \rightarrow+\infty}{\longrightarrow}+\infty$. Hence,

$$
\begin{aligned}
\int_{0}^{t} x^{T}(s) W x(s) d s & \geqslant \sum_{k=1}^{N(t)} \int_{t_{k}}^{t_{k}+\tau} x^{T}(s) W x(s) d s \\
& \geqslant \lambda_{\min }(W) \frac{\beta^{2} \tau}{4} N(t) \underset{t \rightarrow+\infty}{\longrightarrow}+\infty .
\end{aligned}
$$


Additionally, functional (3) admits an upper bound of the form $\left|v\left(x_{t}\right)\right| \leqslant \eta\left\|x_{t}\right\|_{h}^{2} \leqslant \eta G^{2}$, where $\eta=$ const $>0$. Finally,

$v(\varphi)=\underbrace{v\left(x_{t}\right)}_{\geqslant-\eta G^{2}}+\int_{0}^{t} x^{T}(s) W x(s) d s \underset{t \rightarrow+\infty}{\longrightarrow}+\infty$,

which is impossible. Note that we have not used here the second condition of the theorem.

2. We now suppose that the solution $x(t)$ is not uniformly bounded. In this case, one can choose the sequence $\left\{t_{k}\right\}_{k=1}^{\infty}$ so that

$\left\|x\left(t_{k}\right)\right\|=\max _{-h \leqslant t \leqslant t_{k}}\|x(t)\| \underset{k \rightarrow+\infty}{\longrightarrow}+\infty$.

Hence, $x_{t_{k}} \in S$ for every $k$, and

$v(\varphi)=v\left(x_{t_{k}}\right)+\int_{0}^{t_{k}} x^{T}(s) W x(s) d s$

$\geqslant \mu\left\|x\left(t_{k}\right)\right\|^{2} \underset{k \rightarrow+\infty}{\longrightarrow}+\infty$.

The contradiction ends the proof.

Remark 2. Theorem 1 remains true if we replace the set $S$ with the set

$S_{k}=\left\{\varphi:\left\|\varphi^{(l)}(\theta)\right\| \leqslant\left(\sum_{j=0}^{m}\left\|A_{j}\right\|\right)^{l}\|\varphi(0)\|\right.$,

$\theta \in[-h, 0], l=0,1, \ldots, k+1\}$,

where $\varphi^{(l)}$ denotes the lth derivative of $\varphi$, for every natural $k$. The set $S_{k}$ contains $(k+1)$-times continuously differentiable functions $\varphi$.

\section{Method for stability analysis}

In this section, we present a method for investigating the exponential stability of system (1). The main point of the method is to obtain a positive quadratic lower bound on the functional $v$ on the set $S_{k}$ for a given $k$, in accordance with Theorem 1 and Remark 2 . To do this, we provide a numerical scheme based on an approximation of $\varphi \in S_{1}$ by means of a piecewise linear function.

Consider the interval $[-h, 0]=\bigcup_{j=1, \ldots, m}\left[-h_{j},-h_{j-1}\right]$, and divide each of the intervals $\left[-h_{j},-h_{j-1}\right]$ into $N_{j}$ equal parts of the length $\Delta_{j}=\left(h_{j}-h_{j-1}\right) / N_{j}$ by the points

$\theta_{k}^{(j)}=-h_{j-1}-k \Delta_{j}, \quad k=0,1, \ldots, N_{j}, j=1, \ldots, m$.

Let $N=N_{1}+\cdots+N_{m}$ be the total number of intervals in the partition. Consider the function $\varphi \in S_{1}$, and introduce the following notations

$\widehat{\varphi}_{k}^{(j)}=\varphi\left(\theta_{k}^{(j)}\right), \quad k=0,1, \ldots, N_{j}, j=1, \ldots, m ;$

$\widehat{\varphi}^{(j)}=\left(\widehat{\varphi}_{1}^{(j)^{T}}, \ldots, \widehat{\varphi}_{N_{j}}^{(j)^{T}}\right)^{T}, \quad j=1, \ldots, m ;$

$\widehat{\varphi}=\left(\widehat{\varphi}^{(1)^{T}}, \ldots, \widehat{\varphi}^{(m)^{T}}\right)^{T}$.

The dimension of the vector $\widehat{\varphi}$ is $n N$. Additionally, let $p=\varphi(0)=$ $\widehat{\varphi}_{0}^{(1)}$. Then, approximate the function $\varphi \in S_{1}$ by the piecewise linear vector function $\gamma$ :

$\varphi(\theta)=\gamma(\theta)+\eta(\theta), \quad \theta \in[-h, 0]$,

where $\eta$ is the approximation error, and

$\gamma\left(\theta+\theta_{k}^{(j)}\right)=\widehat{\varphi}_{k}^{(j)}+\left(\widehat{\varphi}_{k}^{(j)}-\widehat{\varphi}_{k+1}^{(j)}\right) \frac{\theta}{\Delta_{j}}, \quad \theta \in\left[-\Delta_{j}, 0\right]$,

$k=0, \ldots, N_{j}-1, \quad j=1, \ldots, m$.
Using Taylor's formula and the fact that $\varphi \in S_{1}$, we obtain the following estimation of the error

$\left\|\eta\left(\theta+\theta_{k}^{(j)}\right)\right\| \leqslant \frac{\sqrt{n}}{2}\left(\sum_{l=0}^{m}\left\|A_{l}\right\|\right)^{2}\|p\|\left(\theta^{2}-\theta \Delta_{j}\right)$,

$\theta \in\left[-\Delta_{j}, 0\right], \quad k=0, \ldots, N_{j}-1, j=1, \ldots, m$.

Before substituting expression (4) into functional (3), we divide each of the integrals in the functional into summands according to the partition of the intervals $\left[-h_{j},-h_{j-1}\right]$, and then change the variable in each integral to make it belong to $\left[-\Delta_{j}, 0\right]$. After the substitution, we obtain two groups of summands in the functional. The first group represents functional (3) on the piecewise linear function $\gamma$, without taking the error into account. We denote it by $v_{0}(\cdot)$. The second group includes all the summands with the error. For this one, we apply formula (5) and again use $\varphi \in S_{1}$. As a result, we obtain that the second group admits the estimation of the form $-\delta\|p\|^{2}$, where $\delta=$ const $>0$. Finally, by simple but cumbersome calculations we arrive at the following lower bound for the functional on the set $S_{1}$

$v(\varphi) \geqslant v_{0}(p, \widehat{\varphi}, \mathbf{h}, \mathbf{N})-\delta(\mathbf{h}, \mathbf{N})\|p\|^{2}, \quad \varphi \in S_{1}$,

where $\mathbf{h}=\left(h_{1}, \ldots, h_{m}\right)^{T}, \mathbf{N}=\left(N_{1}, \ldots, N_{m}\right)^{T}$, and $v_{0}(\cdot)$ is a quadratic form of the vector $\left(p^{T}, \widehat{\varphi}^{T}\right)^{T}$. The exact formula for bound (6) in case of scalar equation with one delay is given in Zhabko and Medvedeva (2011).

Introduce the set

$\widehat{S}_{\mathbf{N}}=\left\{\widehat{\varphi}:\left\|\widehat{\varphi}_{k}^{(j)}\right\| \leqslant\|p\|, k=1, \ldots, N_{j}, j=1, \ldots, m\right\}$,

that is the set $S_{1}$ expressed in terms of the vector $\widehat{\varphi}$, and define the function

$z(\mathbf{h}, \mathbf{N})=\min _{\substack{\hat{\varphi} \in \widehat{S}_{\mathbf{N}} \\\|p\|=1}} v_{0}(p, \widehat{\varphi}, \mathbf{h}, \mathbf{N})-\delta(\mathbf{h}, \mathbf{N})$.

We can now rephrase the sufficiency of Theorem 1 as follows.

Theorem 3. If there exists $\mathbf{N}$ such that $z(\mathbf{h}, \mathbf{N})>0$, then system (1) is exponentially stable.

Proof. If there exists $\mathbf{N}$ such that

$\min _{\substack{\widehat{\varphi} \in \widehat{S}_{\mathbf{N}} \\ p \neq \mathbf{0}}}\left(v_{0}(p, \widehat{\varphi}, \mathbf{h}, \mathbf{N})-\delta(\mathbf{h}, \mathbf{N})\|p\|^{2}\right)>0$,

then, by (6), sufficiency of Theorem 1 with the set $S_{1}$ holds. Since $v_{0}-\delta\|p\|^{2}$ is the quadratic form, sign of the latter minimum does not depend on the exact value of $\|p\|$. Therefore, we suppose $\|p\|=$ 1 , and the result follows.

Remark 4. To make a picture complete, we also give the sufficient condition for instability which is based on the results from Zhabko and Medvedeva (2011) and Medvedeva and Zhabko (2013): if there exists $\mathbf{N}$ such that

$\min _{\substack{\widehat{\varphi} \in \widehat{S}_{\mathbf{N}} \\\|p\|=1}} v_{0}(p, \widehat{\varphi}, \mathbf{h}, \mathbf{N})+\delta(\mathbf{h}, \mathbf{N})<0$,

then system (1) is unstable.

To apply the method described in this section to a given system of the form (1), one should try to find $\mathbf{N}$, for which the condition of Theorem 3 or Remark 4 holds. It is worth to mention that the corresponding $\mathbf{N}$ can be found for both exponentially stable and unstable systems, see Lemma 6 for the proof of this fact in the stability case.

Remark 5. In addition to the piecewise linear approximation scheme, a cubic one can be considered by approximating $\varphi \in S_{3}$ 
in each interval of the partition with the cubic vector function

$$
\begin{aligned}
q\left(\theta+\theta_{k}^{(j)}\right)= & g_{1 j}(\theta) \varphi\left(\theta_{k}^{(j)}\right)+g_{2 j}(\theta) \varphi\left(\theta_{k+1}^{(j)}\right) \\
& +g_{3 j}(\theta) \varphi^{\prime}\left(\theta_{k}^{(j)}\right)+g_{4 j}(\theta) \varphi^{\prime}\left(\theta_{k+1}^{(j)}\right), \quad \theta \in\left[-\Delta_{j}, 0\right],
\end{aligned}
$$

instead of $\gamma$. Here

$$
\begin{array}{ll}
g_{1 j}(\theta)=-\frac{2}{\Delta_{j}^{3}} \theta^{3}-\frac{3}{\Delta_{j}^{2}} \theta^{2}+1, & g_{2 j}(\theta)=\frac{2}{\Delta_{j}^{3}} \theta^{3}+\frac{3}{\Delta_{j}^{2}} \theta^{2}, \\
g_{3 j}(\theta)=\frac{1}{\Delta_{j}^{2}} \theta^{3}+\frac{2}{\Delta_{j}} \theta^{2}+\theta, & g_{4 j}(\theta)=\frac{1}{\Delta_{j}^{2}} \theta^{3}+\frac{1}{\Delta_{j}} \theta^{2} .
\end{array}
$$

The main advantage of such approximation is its smoothness, in contrast with the linear one. For details, we refer the reader to Medvedeva and Zhabko (2013).

\section{Convergence issue}

Here we suppose that $h_{j}=\alpha_{j} \mathfrak{h}, j=1, \ldots, m$, where $\alpha_{j}>0$ are fixed, $\mathfrak{h} \geqslant 0$ is a basic delay. Let $\mathbf{h}=\left(\alpha_{1} \mathfrak{h}, \ldots, \alpha_{m} \mathfrak{h}\right)^{T}$. We will use the following notations: $\mathbf{N} \geqslant \mathbf{L}$ means that $N_{j} \geqslant L_{j}, j=1, \ldots, m$, where $\mathbf{N}=\left(N_{1}, \ldots, N_{m}\right)^{T}, \mathbf{L}=\left(L_{1}, \ldots, L_{m}\right)^{T} ; \mathbf{N} \rightarrow+\infty$ means that $N_{j} \rightarrow+\infty, j=1, \ldots, m$. Set matrices $A_{j}, j=0,1, \ldots, m$, and assume that there exists $\mathfrak{h}$ such that system (1) is exponentially stable. Our study of the convergence issue is based on the following lemma.

Lemma 6. Set $\mathfrak{h}$ such that system (1) is exponentially stable. Then there exists $\widetilde{\mathbf{N}}=\left(\widetilde{N}_{1}, \ldots, \widetilde{N}_{m}\right)^{T}$ such that $z(\mathbf{h}, \mathbf{N})>0$ for any $\mathbf{N} \geqslant \widetilde{\mathbf{N}}$.

Proof. The result is based on the fact

$\delta(\mathbf{h}, \mathbf{N}) \underset{\mathbf{N} \rightarrow+\infty}{\longrightarrow} 0$.

By (6) and $v(\varphi) \leqslant v_{0}+\delta$, we have $\left|v(\varphi)-v_{0}\right| \leqslant \delta$, that implies

$v_{0}(p, \widehat{\varphi}, \mathbf{h}, \mathbf{N}) \underset{\mathbf{N} \rightarrow+\infty}{\longrightarrow} v(\varphi)$

for any $\varphi \in S_{1} \cap\{\varphi: \varphi(0)=p,\|p\|=1\}$. Since $v(\varphi)>0$ for exponentially stable system (1), the lemma follows from the definition of limit and formula (7).

Lemma 7. If system (1) is exponentially stable, then there exists $\mu(\mathbf{h})$ $>0$ such that for any given $\mathbf{N}$

$$
\min _{\substack{\widehat{\varphi} \in \widehat{S}_{\mathbf{N}} \\\|p\|=1}} v_{0}(p, \widehat{\varphi}, \mathbf{h}, \mathbf{N}) \geqslant \inf _{\substack{\varphi \in S_{1} \\ \varphi(0)=p \\\|p\|=1}} v(\varphi) \geqslant \mu(\mathbf{h}) .
$$

Proof. The first inequality follows from the fact that the first minimum in (9) is that of the functional $v$ on the set of piecewise linear functions from $S_{1}$, and the second one is the same minimum on a wider set. The second inequality is true for $\mu$ from the necessity part of Theorem 1 .

Remark 8. The necessity part of Theorem 1 gives a constructive way of obtaining $\mu(\mathbf{h})$. If system (1) is not exponentially stable, we can also find $\mu(\mathbf{h})$, but the second inequality in (9) becomes false. The function $\mu(\mathbf{h})$ is continuous and converges to zero only when $\mathfrak{h} \rightarrow+\infty$.

\section{Corollary 9. If for a fixed $\mathbf{h}$ there exists $\mathbf{N}$ such that}

$\min _{\substack{\widehat{\varphi} \in \widehat{S}_{\mathbf{N}} \\\|p\|=1}} v_{0}(p, \widehat{\varphi}, \mathbf{h}, \mathbf{N})<\mu(\mathbf{h})$,

then system (1) is not exponentially stable.

Let $\overline{\mathfrak{h}}_{1}, \overline{\mathfrak{h}}_{2}\left(0<\overline{\mathfrak{h}}_{1}<\overline{\mathfrak{h}}_{2}<+\infty\right)$ be the critical values of basic delay such that system (1) is exponentially stable for $\mathfrak{h} \in\left(\overline{\mathfrak{h}}_{1}, \overline{\mathfrak{h}}_{2}\right)$.
Choose $\tilde{\mathfrak{h}} \in\left(\overline{\mathfrak{h}}_{1}, \overline{\mathfrak{h}}_{2}\right)$, and, in accordance with Lemma 6 , find $\tilde{\mathbf{N}}$ such that $z\left(\alpha_{1} \tilde{\mathfrak{h}}, \ldots, \alpha_{m} \tilde{\mathfrak{h}}, \mathbf{N}\right)>0$ for any $\mathbf{N} \geqslant \tilde{\mathbf{N}}$. For such $\mathbf{N}$, let us define the sequences

$\mathfrak{h}_{\mathbf{N}}^{(1)}=\sup _{\substack{\mathfrak{h}<\tilde{\mathfrak{h}} \\ z(\mathbf{h}, \mathbf{N}) \leqslant 0}} \mathfrak{h}, \quad \mathfrak{h}_{\mathbf{N}}^{(2)}=\inf _{\substack{\mathfrak{h}>\tilde{\mathfrak{h}} \\ z(\mathbf{h}, \mathbf{N}) \leqslant 0}} \mathfrak{h}$.

Since $z(\mathbf{h}, \mathbf{N})$ is a continuous function of $\mathfrak{h} \in\left(\overline{\mathfrak{h}}_{1}, \overline{\mathfrak{h}}_{2}\right)$, it follows from (10) that $z(\mathbf{h}, \mathbf{N})>0$ for $\mathfrak{h} \in\left(\mathfrak{h}_{\mathbf{N}}^{(1)}, \mathfrak{h}_{\mathbf{N}}^{(2)}\right)$ and therefore $\left(\mathfrak{h}_{\mathbf{N}}^{(1)}, \mathfrak{h}_{\mathbf{N}}^{(2)}\right) \subset\left(\overline{\mathfrak{h}}_{1}, \overline{\mathfrak{h}}_{2}\right)$. It means that $\left(\mathfrak{h}_{\mathbf{N}}^{(1)}, \mathfrak{h}_{\mathbf{N}}^{(2)}\right)$ is the interval of exponential stability of system (1) ensured by our method with fixed $\mathbf{N}$.

Theorem 10. Sequences (10) converge, and

$\lim _{\mathbf{N} \rightarrow+\infty} \mathfrak{h}_{\mathbf{N}}^{(1)}=\overline{\mathfrak{h}}_{1}, \quad \lim _{\mathbf{N} \rightarrow+\infty} \mathfrak{h}_{\mathbf{N}}^{(2)}=\overline{\mathfrak{h}}_{2}$.

Proof. We give the proof for the second sequence. For every $\mathbf{N} \geqslant \widetilde{\mathbf{N}}$ we have $\mathfrak{h}_{\mathbf{N}}^{(2)} \in\left[\tilde{\mathfrak{h}}, \overline{\mathfrak{h}}_{2}\right]$, so the sequence is bounded. This means that it has upper and lower limits, and its upper limit is smaller than or equal to $\overline{\mathfrak{h}}_{2}$. Suppose that

$\varliminf_{\mathbf{N} \rightarrow+\infty} \mathfrak{h}_{\mathbf{N}}^{(2)}=\mathfrak{h}_{0}<\overline{\mathfrak{h}}_{2}$.

It implies that we can extract a subsequence

$\left\{\mathfrak{h}_{\mathbf{N}_{\mathbf{j}}}^{(2)}\right\} \subset\left\{\mathfrak{h}_{\mathbf{N}}^{(2)}\right\}$,

where $\mathbf{j}=\left(j_{1}, \ldots, j_{m}\right)^{T}, \mathbf{N}_{\mathbf{j}}=\left(N_{1_{j_{1}}}, \ldots, N_{m_{j_{m}}}\right)^{T}$, such that there exists

$\lim _{\mathbf{j} \rightarrow+\infty} \mathfrak{h}_{\mathbf{N}_{\mathbf{j}}}^{(2)}=\mathfrak{h}_{0}$

Therefore, one can find $\tilde{\mathbf{j}}=\left(\tilde{j}_{1}, \ldots, \tilde{j}_{m}\right)^{T}$ such that for every $\mathbf{j} \geqslant \tilde{\mathbf{j}}$ system (1) with $\mathfrak{h}=\mathfrak{h}_{\mathbf{N}_{\mathbf{j}}}^{(2)}$ is exponentially stable. Denote $\mathbf{h}_{\mathbf{N}_{\mathbf{j}}}=\left(\alpha_{1} \mathfrak{h}_{\mathbf{N}_{\mathbf{j}}}^{(2)}, \ldots, \alpha_{m} \mathfrak{h}_{\mathbf{N}_{\mathbf{j}}}^{(2)}\right)^{T}$. On the other hand, by (10) and the continuity of $z$ on the exponential stability interval, we have $z\left(\mathbf{h}_{\mathbf{N}_{\mathbf{j}}}, \mathbf{N}_{\mathbf{j}}\right) \leqslant 0$. Hence,

$\min _{\substack{\widehat{\varphi} \in \widehat{S}_{\mathbf{N}_{\mathbf{j}}} \\\|p\|=1}} v_{0}\left(p, \widehat{\varphi}, \mathbf{h}_{\mathbf{N}_{\mathbf{j}}}, \mathbf{N}_{\mathbf{j}}\right) \leqslant \delta\left(\mathbf{h}_{\mathbf{N}_{\mathbf{j}}}, \mathbf{N}_{\mathbf{j}}\right) \underset{\mathbf{j} \rightarrow+\infty}{\longrightarrow} 0$,

which is due to (8) and $\mathfrak{h}_{\mathbf{N}_{\mathbf{j}}}^{(2)} \underset{\mathbf{j} \rightarrow+\infty}{\longrightarrow} \mathfrak{h}_{0} \in \mathbb{R}$. Additionally, according to Remark 8,

$\mu\left(\mathbf{h}_{\mathbf{N}_{\mathbf{j}}}\right) \underset{\mathbf{j} \rightarrow+\infty}{\longrightarrow} \mu\left(\alpha_{1} \mathfrak{h}_{0}, \ldots, \alpha_{m} \mathfrak{h}_{0}\right)>0$

This means that there exists $\overline{\mathbf{j}} \geqslant \tilde{\mathbf{j}}$ such that for $\mathbf{h}_{\mathbf{N}_{\overline{\mathbf{j}}}}$ the inequality from Corollary 9 holds, which contradicts the exponential stability of system (1). Therefore, assumption (11) is false, and the theorem is proved for the sequence $\left\{\mathfrak{h}_{\mathbf{N}}^{(2)}\right\}$. The proof for $\left\{\mathfrak{h}_{\mathbf{N}}^{(1)}\right\}$ is similar.

The significance of Theorem 10 lies in the fact that the interval of exponential stability ensured by our method converges "from the inside" to the exact one as $\mathbf{N} \rightarrow+\infty$. We call this property the convergence of the method.

Remark 11. In slightly different terms, Theorem 10 remains valid if $\overline{\mathfrak{h}}_{1}=0$ or $\overline{\mathfrak{h}}_{2}=+\infty$. Indeed, $\overline{\mathfrak{h}}_{1}=0$ means that the system is exponentially stable for $\mathfrak{h}=0$, and therefore for small basic delays. In this case, we can take $\tilde{\mathfrak{h}}=0$ and consider only the second sequence. If $\overline{\mathfrak{h}}_{2}=+\infty$, then $\left\{\mathfrak{h}_{\mathbf{N}}^{(2)}\right\}$ may include the elements equal to $+\infty$. In this case, we suppose its limit to be equal to $+\infty$. With such redefinition, the convergence implies $\mathfrak{h}_{\mathbf{N}}^{(2)} \underset{\mathbf{N} \rightarrow+\infty}{\longrightarrow}+\infty$. Finally, if system (1) is not exponentially stable for any basic delay, then $z(\mathbf{h}, \mathbf{N}) \leqslant 0$ for any $\mathbf{h}$ and $\mathbf{N}$. 


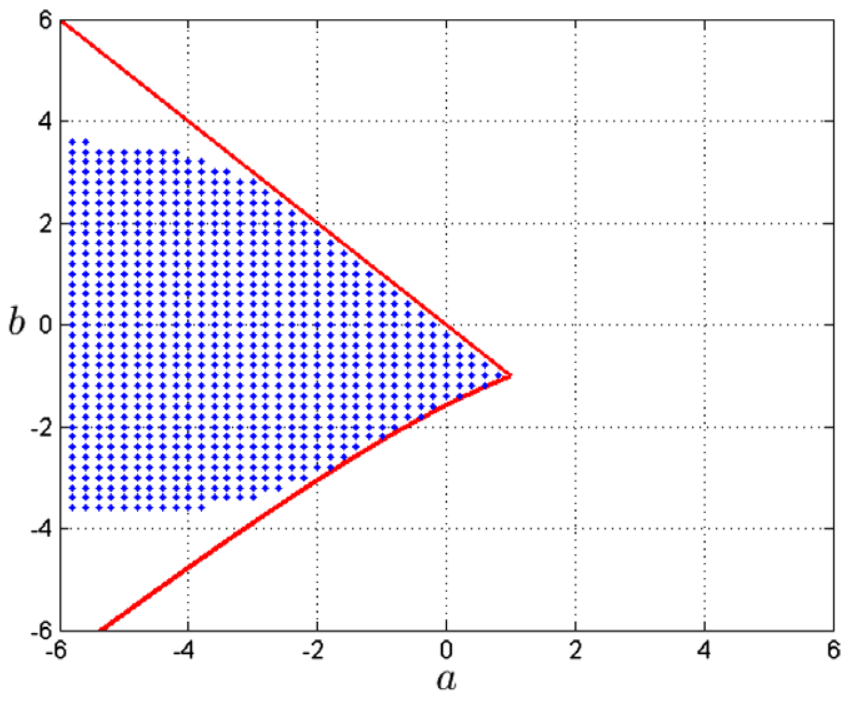

Fig. 1. Eq. (12), $N=10$, cubic approximation.

\section{Examples}

In this section, we provide two examples to illustrate the application of the method described in Section 4 and its convergence (see Section 5). In each example, we compare the exact stability domain that is known with the domain obtained by verification of Theorem 3, for the case of linear or cubic spline approximations. In the figures, the curves depict the boundaries of the exact exponential stability domains in the space of parameters $a$ and $b$ as well as the points correspond to the pairs $(a, b)$ for which Theorem 3 with fixed $\mathbf{N}$ holds.

To check the condition of Theorem 3, one needs to calculate the value of function (7) which depends on the Lyapunov matrix $U(\tau)$, $\tau \in[0, h]$. We compute it for $W=I$, using the "semianalytic" method proposed in Garcia-Lozano and Kharitonov (2004) where system (2) is reduced to a system of ordinary differential equations with some boundary conditions. This method is applicable only in case of commensurate delays, whereas, when the delays are incommensurate, the only approximations of the matrix $U$ are available, see Kharitonov (2013).

Notice that in calculations we replace the norms used for the vectors in the original set $\widehat{S}_{\mathbf{N}}$ (or in the analogous set for the cubic approximation scheme) by the absolute values of their components. Theorem 3 remains true with such modification.

Example 12. Consider a scalar equation with one delay

$\dot{x}(t)=a x(t)+b x(t-h), \quad a, b=$ const, $h>0$.

It is well-known (see, for instance, Bellman \& Cooke, 1963, p. 444) that, for a given $h$, Eq. (12) is exponentially stable if and only if

$a<1 / h$ and $a<-b<\omega / \sin (\omega h)$,

where $\omega$ is a root of the equation $a=\omega \cot (\omega h)$ such that $0<$ $\omega<\pi / h$ and $\omega=\pi / 2 h$ for $a=0$. Fig. 1 shows that, for $h=1$, the stability domain obtained by our method with $N=10$ and the cubic approximation scheme is a good estimation for the exact one.

Suppose, that Eq. (12) is exponentially stable for $h=0$, and denote its unique critical delay by $\bar{h}$, which is finite or takes the value $+\infty$. Consider two problems that are inverse to each other.

Problem 13. For fixed $a, b$, and $N$, find with an accuracy 0.001 the maximal value $h_{N}$ such that Theorem 3 holds for $h \in\left[0, h_{N}\right]$.

Problem 14. For fixed $a, b$, and $h<\bar{h}$, find $N$ such that Theorem 3 holds.
Table 1

Problem 13: the values $h_{N}$.

\begin{tabular}{|c|c|c|c|c|c|c|}
\hline \multirow{2}{*}{$\begin{array}{l}a, b, \\
N \\
\text { Type }\end{array}$} & \multicolumn{2}{|c|}{$\begin{array}{l}a=-1 \\
b=-1\end{array}$} & \multicolumn{2}{|c|}{$\begin{array}{l}a=-0.5 \\
b=-1\end{array}$} & \multicolumn{2}{|c|}{$\begin{array}{l}a=0, \\
b=-1\end{array}$} \\
\hline & Linear & Cubic & Linear & Cubic & Linear & Cubic \\
\hline$N=1$ & 0.499 & 0.772 & 0.562 & 0.934 & 0.657 & 1.227 \\
\hline$N=5$ & 1.308 & 2.334 & 1.371 & 2.184 & 1.448 & 1.5707 \\
\hline$N=10$ & 1.834 & 3.663 & 1.736 & 2.388 & 1.5707 & 1.5707 \\
\hline$N=20$ & 2.530 & 5.63 & 2.068 & 2.417 & 1.5707 & 1.5707 \\
\hline $\bar{h}$ & \multicolumn{2}{|c|}{$+\infty$} & \multicolumn{2}{|c|}{$\approx 2.418$} & \multicolumn{2}{|c|}{$\approx 1.5708$} \\
\hline
\end{tabular}

Table 2

Problem 14: the values $N_{1}$ and $N_{3}$.

\begin{tabular}{lrrllllll}
\hline \multicolumn{2}{l}{$a=-1, b=-1$} \\
$h$ & 1 & 2 & 2.5 & 3 & 3.5 & 4 & 4.5 & 5 \\
$N_{1}$ & 3 & 13 & 20 & 30 & 42 & 56 & 74 & 95 \\
$N_{3}$ & 2 & 4 & 6 & 8 & 10 & 12 & 15 & 17 \\
\hline$a=$ & $-0.5, b=$ & -1 & & & & & & \\
\hline$h$ & 1 & 1.5 & 1.9 & 2.1 & 2.3 & 2.4 & 2.415 & 2.417 \\
$N_{1}$ & 3 & 7 & 14 & 22 & 43 & 117 & 260 & 393 \\
$N_{3}$ & 2 & 2 & 4 & 5 & 7 & 12 & 18 & 20 \\
\hline
\end{tabular}

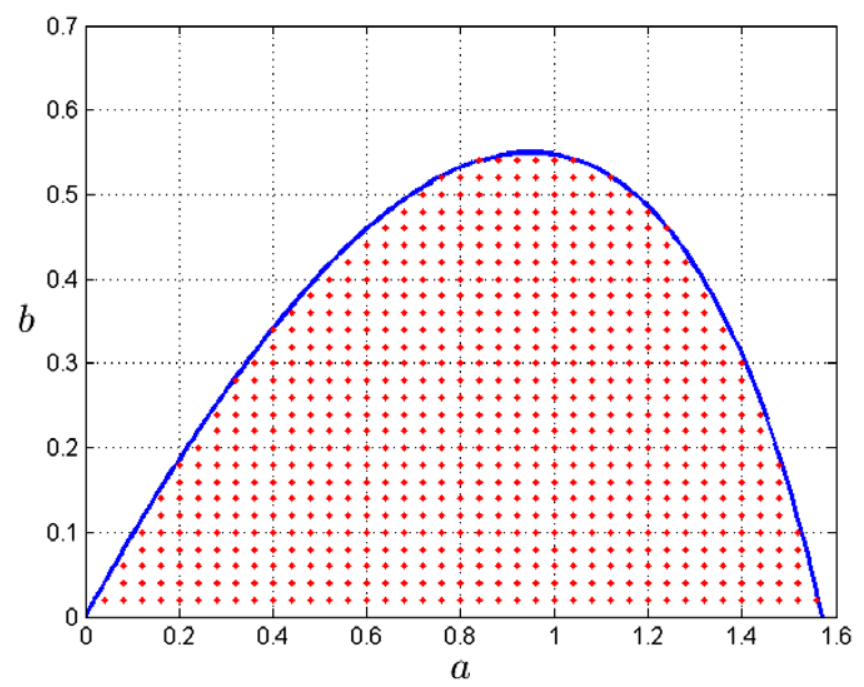

Fig. 2. System (13), $N=10$, cubic approximation

For different equations of the form (12), the solutions of Problems 13 and 14 are presented in Tables 1 and 2 respectively. In Table 2, $N=N_{1}$ corresponds to the linear approximation scheme while $N=N_{3}$ corresponds to the cubic one. It is clear that the results obtained by the cubic approximation are much better than those obtained by the linear one due to the smoothness of the first one in contrast to the latter. Nevertheless, Table 1 illustrates the convergence of the method for both types of approximation.

Example 15. Consider the equation

$\ddot{y}(t)+a \dot{y}(t-1)+b y(t-1)=0, \quad a, b=$ const,

which is equivalent to the following system

$\dot{x}(t)=\left(\begin{array}{ll}0 & 1 \\ 0 & 0\end{array}\right) x(t)+\left(\begin{array}{cc}0 & 0 \\ -b & -a\end{array}\right) x(t-1)$.

It is known (see Bellman \& Cooke, 1963, p. 450) that the exact domain of exponential stability of system (13) in the space of parameters $a$ and $b$ is described by the conditions $0<a<\pi / 2$ and $0<b<\omega^{2} \cos \omega$, where $\omega$ is a root of the equation $a=\omega \sin \omega$ such that $0<\omega<\pi / 2$. This domain, along with that obtained by our method with $N=10$ and the cubic approximation scheme, is depicted on Fig. 2. 


\section{Concluding remarks}

In this paper, a necessary and sufficient stability condition joining the Razumikhin and the Lyapunov-Krasovskii approaches is provided for linear systems with several delays. The condition allows to estimate the stability domains in the parameter space.

\section{Acknowledgments}

The authors thank Prof. V.L. Kharitonov, the reviewers, and the Associate Editor for their valuable comments and suggestions that helped us to improve the presentation of the manuscript.

\section{References}

Bellman, R., \& Cooke, K. L. (1963). Differential-difference equations. New York: Academic Press.

Egorov, A., \& Mondie, S. (2013). Necessary conditions for the stability of multiple time-delay systems via the delay Lyapunov matrix. In Proceedings of 11th IFAC workshop on time-delay systems, Grenoble, France (pp. 12-17).

Garcia-Lozano, H., \& Kharitonov, V. L. (2004). Lyapunov matrices for time delay system with commensurate delays. In Proceedings of the 2nd symposium on system, structure and control, Oaxaca, Mexico.

Huang, W. (1989). Generalization of Liapunov's theorem in a linear delay system. Journal of Mathematical Analysis and Applications, 142, 83-94.

Infante, E. F., \& Castelan, W. B. (1978). A Liapunov functional for a matrix differencedifferential equation. Journal of Differential Equations, 29, 439-451.

Kharitonov, V. L. (2013). Time-delay systems: Lyapunov functionals and matrices. Basel: Birkhäuser.

Kharitonov, V. L., \& Niculescu, S. I. (2003). On the stability of linear systems with uncertain delay. IEEE Transactions on Automatic Control, 48, 127-132.

Kharitonov, V. L., \& Zhabko, A. P. (2003). Lyapunov-Krasovskii approach to the robust stability analysis of time-delay systems. Automatica, 39, 15-20.

Krasovskii, N. N. (1956). On the second Lyapunov method application to the equations with delay. Prikladnaya Matematikai Mekhanika, 20, 315-327. (in Russian)
Medvedeva, I. V., \& Zhabko, A. P. (2013). Constructive method of linear systems with delay stability analysis. In Proceedings of 11th IFAC workshop on time-delay systems, Grenoble, France (pp. 1-6).

Ochoa, G., Mondie, S., \& Kharitonov, V. L. (2009). Time delay systems with distributed delays: Critical values. In Proceedings of 8th IFAC workshop on timedelay systems, Sinaia, Rumania.

Razumikhin, B. S. (1956). On the stability of time-delay systems. Prikladnaya Matematikai Mekhanika, 20,500-512. (in Russian).

Repin, Y. M. (1965). Quadratic Lyapunov functionals for systems with delay. J. Applied Mathematics and Mechanics, 29, 669-672. (translation of Prikl. Mat. Mekh., 29, 1965, pp 564-566).

Zhabko, A. P., \& Medvedeva, I. V. (2011). The algebraic approach to stability analysis of differential-difference systems. Vestnik Saint-Petersburg State University, 1, 9-20. (in Russian).

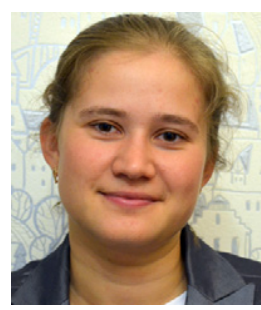

Irina V. Medvedeva received the M.Sc. degree in Mathematics in 2011, from St.-Petersburg State University, St.Petersburg, Russia, where she is now working on her Ph.D. thesis at the Department of Control Theory. She is currently a Lecturer at the Department of Mechanics of Controlled Motion at the same University. Her research interests include time-delay systems, stability and robust stability.

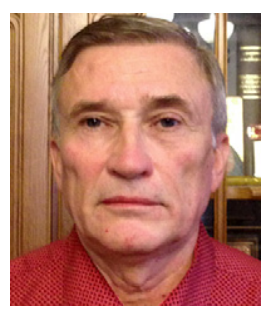

Alexey P. Zhabko received the M.A. Degree in 1973, the Candidate of Science Degree in Automatic Control in 1981, and the Doctor of Science Degree in Automatic Control in 1992, all from the Leningrad State University, Russia. Dr. Zhabko is currently the Head of the Control Theory Department at the Faculty of Applied Mathematics and Control Processes, St.-Petersburg State University, St.Petersburg, Russia. His research interests include control theory, time-delay systems, stability, identification and robust control. 